\title{
Influencia de un carenado superior en la fuerza de
} arrastre de un camión

\author{
Influence of a higher fairing on the pulling force of a truck
}

David Paul Pachacama Gualotuña. ${ }^{1}$, Darwin Anderson Pachacama Gualotuña. ${ }^{2}$, Claudio Constante Córdova Orellana. ${ }^{3}$ \& Fredy Rafael Llulluna Llumiquinga. ${ }^{4}$

\begin{abstract}
.
DOI: https://doi.org/10.33262/cienciadigital.v5i2.1610

Introduction: The consumption of diesel fuel in the country represented $36.61[\%]$ of the demand for petroleum derivatives in 2019 , which is why it seeks to propose savings alternatives in the land freight transport sector. For this reason, the influence of an upper cabin fairing (windbreaker) on the drag force, which is generated when the truck moves forward, displacing a large amount of air that flows through the exterior and interior of the truck, was analyzed. Objective: To analyze the influence of a higher fairing on the drag force of a truck using the one-dimensional equation of the drag force and computational fluid dynamics software. Methodology: This effect was estimated using the one-dimensional drag force equation, which relates the drag coefficient, air density, frontal area, air speed and truck speed, with the help of a software of computational fluid dynamics (CFD), which requires a simplified geometry at 1: 1 scale of the truck and the upper fairing, the domain is generated and the boundary conditions governing the physical phenomenon are taken. Results: A decrease of $0.102[\mathrm{kN}]$ is obtained at a truck speed of $40[\mathrm{~km} / \mathrm{h}]$, as the speed increases to $120[\mathrm{~km} / \mathrm{h}]$, the force decreases by 0.788 $[\mathrm{kN}]$. Conclusions: There is a reduction of $8.734[\%]$ in the drag force when using the

1 Universidad de las Fuerzas Armadas ESPE, Departamento de Ciencias Exactas, Quito, Ecuador, dppachacama1@espe.edu.ec, ORCID: https://orcid.org/0000-0003-4979-7332

2 Universidad de las Fuerzas Armadas ESPE, Departamento de Ciencias Exactas, Quito, Ecuador, dapachacama@espe.edu.ec, ORCID: https://orcid.org/0000-0003-1408-8261

3 Escuela Politécnica Nacional, Departamento de Formación Básica, Quito, Ecuador, claudio.cordova@epn.edu.ec, ORCID: https://orcid.org/0000-0002-6972-183X

4 Universidad Regional Amazónica Ikiam, Facultas de Ciencias Socioambientales, Tena, Ecuador, fredy.1lulluna@ikiam.edu.ec, ORCID: https://orcid.org/0000-0001-5981-2394
\end{abstract}


upper cabin fairing, this is complemented by the study carried out on fuel consumption on the road where there is a saving of 4.63 [\%] with the use of the upper cabin fairing.

Keywords: Drag force; drag coefficient; upper fairing; computational fluid dynamics; one-dimensional equation; air density.

\section{Resumen.}

Introducción: El consumo del combustible diésel en el país representó un 36.61 [\%] de la demanda de derivados de petróleo en el año 2019, razón por la cual se busca proponer alternativas de ahorro en el sector del transporte terrestre de carga. Por esta razón se analizó la influencia de un carenado superior de cabina (rompe-viento) en la fuerza de arrastre, que se genera cuando el camión avanza desplazando gran cantidad de aire que fluye por el exterior e interior del camión. Objetivo: Analizar la influencia de un carenado superior en la fuerza de arrastre de un camión mediante la ecuación unidimensional de la fuerza de arrastre y un software de dinámica de fluidos computacional. Metodología: Este efecto se estimó mediante la ecuación unidimensional de la fuerza de arrastre, la cual relaciona el coeficiente de arrastre, la densidad del aire, el área frontal, la velocidad del aire y la velocidad del camión, con la ayuda de un software de dinámica de fluidos computacional (CFD), el cual requiere una geometría simplificada a escala 1:1 del camión y el carenado superior, se genera el dominio y se toma las condiciones de frontera que rige el fenómeno físico. Resultados: Se obtiene una disminución del $0.102[\mathrm{kN}]$ a una velocidad del camión de $40[\mathrm{~km} / \mathrm{h}]$, conforme se incrementa la velocidad a $120[\mathrm{~km} / \mathrm{h}]$, la fuerza disminuye en 0.788 [kN]. Conclusiones: Se tiene una reducción del 8.734 [\%] en la fuerza de arrastre al utilizar en carenado superior de cabina, esto se complementa con el estudio realizado de consumo de combustible en carretera en donde se tiene un ahorro del 4.63 [\%] con el uso del carenado superior de cabina.

Palabras claves: Fuerza de arrastre; coeficiente de arrastre; carenado superior; dinámica de fluidos computacional; ecuación unidimensional; densidad del aire.

\section{Introducción.}

El consumo interno de combustible diésel 2 y el diésel premiun que se utiliza en el transporte terrestre de carga, tuvo un valor de 32,945 millones de barriles, frente a las gasolinas que se utiliza en el transporte liviano que tuvo un valor de 30,156 millones de barriles, han hecho que varias instituciones públicas y privadas emprendan estudios relacionados a la eficiencia energética en el sector automotriz con la finalidad de reducir el consumo de combustible y por ende las emisiones contaminantes que emiten los vehículos de combustión interna (Petroecuador, 2019). 
Según datos del balance energético nacional, el consumo de 43 millones de barriles equivalentes de petróleo se lo lleva en sector del transporte, el terrestre con un 87 [\%], el aéreo con un $6[\%]$ y el marítimo con un 7 [\%], dentro del transporte terrestre ocupa un 44 [\%] la carga pesada, 20 [\%] la carga liviana, 15 [\%] autos y jeeps, 3 [\%] buses, 3 [\%] taxis y 2 [\%] otros. En diesel se han consumido 819,18 miles de galones, de los cuales la carga pesada representa un 74 [\%], la carga liviana 19 [\%], pasajeros colectivo 6 [\%] y pasajeros individual 1 [\%] (Balance-Energético, 2016).

La venta de camiones registra un valor de 150.952 unidades en los últimos 19 años a partir del año 2.000, gran parte de estos camiones carecen de un carenado (rompe-vientos) colocado en la parte superior de la cabina del camión, sea este un accesorio proporcionado por la casa fabricante o construido bajo especificaciones del propietario en una empresa de carrocerías (AEADE, 2019).

La aerodinámica es una ciencia aplicada con varias aplicaciones prácticas en ingeniería. No importa cuán elegante pueda ser una teoría aerodinámica, o matemáticamente compleja puede ser una solución numérica, o cuán sofisticado es un experimento aerodinámico, todos estos esfuerzos suelen estar dirigidos a la predicción de fuerzas momentos, la transferencia de calor a los cuerpos que se mueven a través de un fluido (generalmente aire) y a la determinación de flujos que se mueven internamente a través de conductos (Jhon D., 1991).

Estudios realizados en la ciudad de Cuenca de un vehículo liviano mediante un software de dinámica de fluidos computacional, obtienen un incremento del coeficiente de arrastre de 0,293 a un valor de 0,318 con el análisis de la influencia de llevar las ventanas totalmente abiertas en el vehículo, resultado obtenido bajo condiciones meteorológicas de la zona (presión atmosférica $74,5 \mathrm{kPa}$ y densidad del aire $0,957 \mathrm{~kg} / \mathrm{m}^{3}$ ) y una altitud de 2.550 m.s.n.m. (Aguilar, Caldas, Rivera, \& Tapia, 2017).

Un modelo a escala 1:32 de un tracto camión con su zona de carga se someten a un túnel de viento por separado, obteniendo un coeficiente de arrastre de 0,608 correspondiente solo a la cabina del tracto camión, en cambio cuando se adiciona la zona de carga al tracto camión el coeficiente de arrastre se incrementa a un valor de 0,704 también se visualiza el flujo de aire que circula en los dos casos de estudio, un de las características del túnel de viento es variar la velocidad del viento en un rango de $0[\mathrm{~m} / \mathrm{s}]$ a $28[\mathrm{~m} / \mathrm{s}](0 \mathrm{~km} / \mathrm{h}$ a 100,8 km/h) (Bayindirli, Salman, \& Akansu, 2016)

El coeficiente de arrastre es un numero adimensional que varía según el diseño exterior e interior, visibilidad, comodidad, estilo, estabilidad, etc. de las superficies de la cabina y zona de carga, adquiere un valor de 0,78 en camiones sin la ayuda dispositivos aerodinámicos y se reduce a 0,64 cuando se utiliza un carenado superior de cabina (SAEJ2188, 2018). Estudios realizados en USA indican que se logra una reducción del 15 [\%] en el coeficiente de arrastre de un tracto-camión y un 6,5 [\%] en el consumo de combustible (Hirz \& Stadler, 2013). 
Al evaluar el coeficiente de arrastre en un software de dinámica de fluidos computacional (CFD) de un semirremolque cisterna, bajo diferentes condiciones atmosféricas en la provincia de Riobamba se obtuvo 1,18 y en la provincia del Guayas el valor fue de 0,86 estos resultados son adimensionales, esto es debido a la presión atmosférica que varía según la altura donde se realice las pruebas, a nivel del mar el flujo de aire que recorre por el vehículo restringe considerablemente su movimiento en relación a un punto con altura (Remache, Tipanluisa, Salvador, \& Erazo, 2015).

Estudios realizados en Chile bajo la norma SAE J1321 la cual estipula un procedimiento de medición del consumo de combustible en camiones, para ello se requiere recorrer una distancia mayor a $64[\mathrm{~km}]$, con una velocidad constante de $90[\mathrm{~km} / \mathrm{h}]$, la medición de la cantidad de combustible se la realiza al inicio y final del recorrido mediante el método de gravimetría (pesar el combustible en una balanza), la diferencia entre estos valores dará como resultado el consumo de combustible, adicional se debe cumplir con varios parámetros tanto para el camión, tramo de carretera y condiciones atmosféricas. Al comparar las cabinas de un tracto-camión Freigthliner (Argosy y Columbia) se obtiene un 3,96 [\%] en el ahorro de combustible por el diseño aerodinámico que posee la cabina Columbia del tracto-camión (Villalobos, Gavilan, Salazar, \& Rojas, 2012). Otro estudio realizado bajo esta misma normativa, se obtiene como resultado que al utilizar varios dispositivos aerodinámicos (carenado superior, frontal y lateral, generador de vórtices y cubre tanque) se logra una reducción del 15.4 [\%] del consumo de diesel de un tractocamión (Villalobos, Arancibia, Retamal, Olivo, \& Vásquez, 2011).

En Ecuador el consumo de combustible de un camión al utilizar un deflector de aire (carenado superior) bajo la norma DIN 70 030-2 la cual también estipula en su procedimiento recorrer una distancia de $10 \mathrm{~km}$, con una velocidad constante de $90 \mathrm{~km} / \mathrm{h}$, la medición de la cantidad de combustible se la realiza al inicio y final del recorrido mediante el método de gravimetría, la diferencia entre estos valores dará como resultado el consumo de combustible, adicional se debe cumplir con varios parámetros tanto para el camión, tramo de carretera y condiciones atmosféricas. Al realizar las pruebas en la carretera del territorio se obtuvo un ahorro de 4.63 [\%] para un camión de carga mediana (Pachacama \& Simbaña, 2017).

\section{Metodologia.}

Se analizó un camión estándar de carga mediana, con su respectiva zona de carga y carenado superior de cabina fabricados bajo especificaciones del propietario, bajo la ecuación unidimensional de la fuerza de arrastre, de manera que se puedan observar los cambios que se generan, al variar los parámetros que la componen. Además, se utiliza un software de dinámica de fluidos computacional (CFD) para estimar la fuerza de arrastre bajo condiciones de operación, del fenómeno físico que se produce con el desplazamiento del camión y su entorno.

El vehículo escogido se ha destacado durante varios años, por sus ventas específicamente en chasis para buses y camiones, en el año 2019 se posiciono con 4210 unidades vendidas, 
ocupando un 3.18 [\%] de participación de mercado en este sector, en relación a sus competidores, en la tabla 1 se indica varias especificaciones del camión seleccionado (AEADE, 2019).

Tabla 1. Características del camión.

\begin{tabular}{ll}
\hline \multicolumn{1}{c}{ Descripción } & \multicolumn{1}{c}{ Valor } \\
\hline Marca & HINO 500 \\
Modelo & GD8JLSA \\
Peso bruto vehicular & $11900 \mathrm{~kg}$ \\
Potencia & $191 \mathrm{~kW} \mathrm{@} \mathrm{2500} \mathrm{rpm}$ \\
Torque & $745 \mathrm{Nm} \mathrm{@} \mathrm{1500} \mathrm{rpm}$ \\
Velocidad máxima & $121 \mathrm{~km} / \mathrm{h}$ \\
Pendiente superable & $47.4 \%$ \\
Capacidad del tanque & $200 \mathrm{~L}$ \\
Sistema de combustible & Inyección directa \\
\hline
\end{tabular}

Fuente: (Hino-Motors, 2014)

La fuerza resistiva ocasionada por el flujo de aire generado cuando el vehículo avanza hacia adelante por una carretera, se la puede estimar mediante la ecuación unidimensional (Jhon D., 1991).

$$
L=C_{D} \cdot q_{\infty} \cdot S
$$

En donde:

$L \quad$ : Fuerza de arrastre $(\mathrm{N})$

$C_{D}$ : Coeficiente de arrastre (adimensional)

$q_{\infty}$ : Presión dinámica $(\mathrm{kg} / \mathrm{m})$

$S \quad$ : Área frontal $\left(\mathrm{m}^{2}\right)$

Presión dinámica de corriente libre de aire, es la energía cinética del fluido debida a la velocidad del fluido en su movimiento. Esta presión no se manifiesta ejerciendo una fuerza sobre una superficie, como ocurre con la presión estática, sino que es la energía por unidad de volumen que posee el fluido en movimiento y se puede calcular mediante la ecuación (Ehsani, Gao, Gay, \& Emadi, 2014).

$$
q_{\infty}=\frac{1}{2} \rho_{\infty}\left(v+v_{\infty}\right)^{2}
$$

En donde:

$\rho_{\infty}:$ Densidad del aire $\left(\mathrm{kg} / \mathrm{m}^{3}\right)$

$v$ : Velocidad del vehículo $(\mathrm{m} / \mathrm{s})$ 
$v_{\infty}:$ Velocidad del aire $(\mathrm{m} / \mathrm{s})$

Al utilizar el método de dinámica de fluidos computacional (CFD) para estimar el fenómeno físico que está ocurriendo con el aire al momento de circular por la superficie del camión, se requieren varias etapas de análisis como se indica en la figura 1 (Hirsch, 2007).

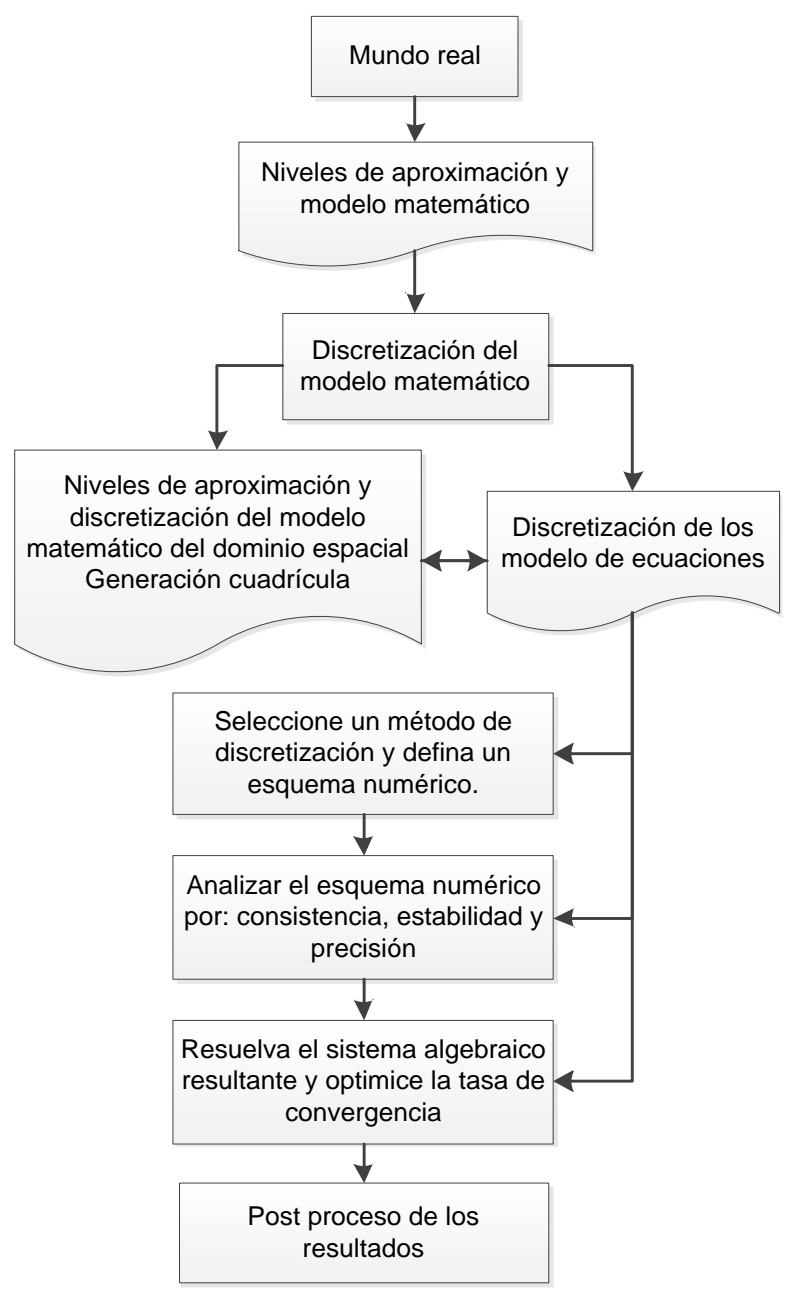

Figura 1. Estructura de un sistema de simulación CFD

Fuente: (Hirsch, 2007)

El software ANSYS es uno de los simuladores que emplea el método de elementos finitos (FEM) y volúmenes finitos (FVM) aplicada a una malla, uno de los módulos para el estudio de flujos es el CFX en el cual se pude modelar: flujos estacionarios y transitorios; flujo laminar y turbulento; flujos subsónicos, transónicos y supersónicos; transferencia de calor y radiación térmica, etc. Su estructura se indica en la figura 2 (Ansys, 2018). 


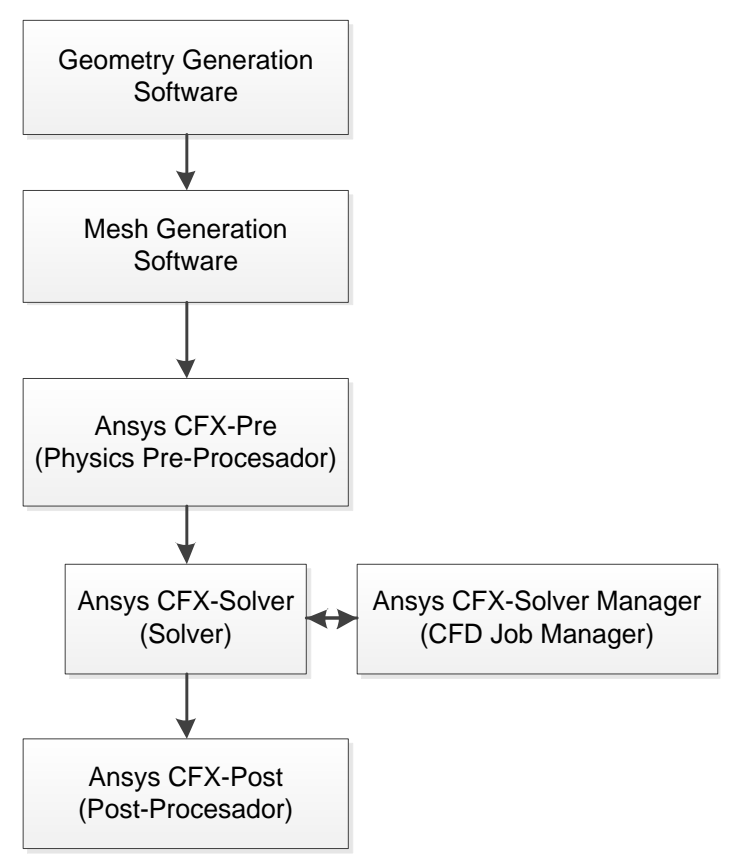

Figura 2. Estructura del módulo CFX

Fuente: (Ansys, 2018)

Las ecuaciones de continuidad que ocupa el módulo CFX son las siguientes:

- Ecuación de continuidad de la masa

$$
\frac{\partial}{\partial t}\left(\gamma \alpha_{q} \rho_{q}\right)+\nabla *\left(\gamma \alpha_{q} \rho_{q} \overrightarrow{v_{q}}\right)=\gamma \sum_{\substack{p=1 \\ P \neq q}}^{n}\left(m_{p q}-m_{q p}\right)+\gamma S_{q}
$$

Dónde:

$\gamma \quad$ : Porosidad

$\rho_{q}:$ Densidad de flujo

$\alpha_{q}$ : Volumen fraccional del fluido

$\overrightarrow{v_{q}}$ : Vector velocidad del fluido

$S_{q}:$ Término fuente

$m_{p q} ; m_{q p}:$ Transferencia de masa entre los puntos o fases p-q y q-p

- Ecuación de continuidad de la energía

$$
\begin{gathered}
\frac{\partial}{\partial t}\left(\alpha_{q}\left(\gamma \rho_{q} h_{q}+(1-\gamma) \rho_{s} h_{s}\right)\right)+\nabla *\left(\gamma \alpha_{q} \rho_{q} \overrightarrow{v_{q}} h_{q}\right)=-\gamma \alpha_{q} \frac{\partial p_{q}}{\partial t}+ \\
\gamma \overline{\bar{\tau}}_{q}: \nabla \overrightarrow{v_{q}}-\nabla *\left(\alpha_{q}\left(\gamma k_{q}+(1-\gamma) k\right)\right)+ \\
\gamma S_{q}+\gamma \sum_{p=1}^{n}\left(Q_{p q}+\dot{m}_{p q} h_{p q}-\dot{m}_{q p} h_{q p}\right)
\end{gathered}
$$


Dónde:

$p ; q$ : Punto o fase

$s \quad$ : Material sólido

$h \quad$ : Entalpia

$k \quad$ : Conductividad térmica

$Q_{p q}$ : Transferencia de calor entre la fase p-q

$\rho \quad$ : Densidad del fluido

$S \quad$ : Fuente de calor

$\alpha \quad$ : Fase de fracción de volumen

$\vec{v} \quad:$ Vector velocidad

$h_{p q} ; h_{q p}$ : Diferencia de la entalpia total entre la fase $\mathrm{p}-\mathrm{q}$ y q-p

- Ecuación de la cantidad de movimiento (Navier Stokes)

$$
H(v-\vec{v})+(1-H)(-\nabla p+\nabla * T+\rho g-\rho a)=0
$$

Dónde:

$H$ : Función de paso

$v$ : Velocidad

$\vec{v} \quad$ : Velocidad local de la parte móvil

$p$ : Presión del fluido

$T$ : Tensor de corte

$\rho g$ : Fuerza de volumen

$\rho a:$ Termino de la aceleración

\section{Resultados.}

Se establece una ubicación dentro de las regiones del ecuador (litoral, sierra, amazonia) que disponga de estaciones de monitoreo atmosférico como se indica en la figura 3, por lo tanto se considera el corredor vial estatal Santo Domingo - La Concordia, en donde previamente se realizó un estudio relacionado al consumo de combustible (INAMHI, 2020).

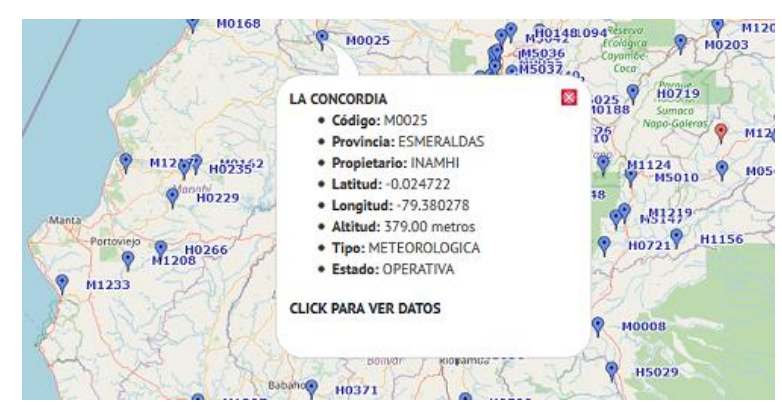

Figura 3. Estación meteorológica M0025

Fuente: (INAMHI, 2020) 
En la tabla 2 se indican los parámetros atmosféricos.

Tabla 2. Parámetros atmosféricos M0025

\begin{tabular}{lc}
\hline \multicolumn{1}{c}{ Descripción } & Valor \\
\hline Fecha - hora & $21-07-2020$ \\
& $13: 00: 00$ \\
Humedad relativa del aire & $85 \%$ \\
Precipitación & $0 \mathrm{~mm}$ \\
Presión atmosférica & $985 \mathrm{hPa}$ \\
Temperatura del aire & $25{ }^{\circ} \mathrm{C}$ \\
Velocidad del viento & $1.4 \mathrm{~m} / \mathrm{s}$ \\
Densidad del aire & $1.1348714 \mathrm{~kg} / \mathrm{m}^{3}$ \\
(CENAM, 2020)
\end{tabular}

Fuente: Elaboración propia

El área frontal proyectada del camión tiene un valor de $8.223\left[\mathrm{~m}^{2}\right]$ se la obtiene mediante el modelo simplificado a escala 1:1 de un software de diseño mecánico, como se indica en la figura 4, este modelo se utiliza en CFD por el gasto computacional (horas de simulación).

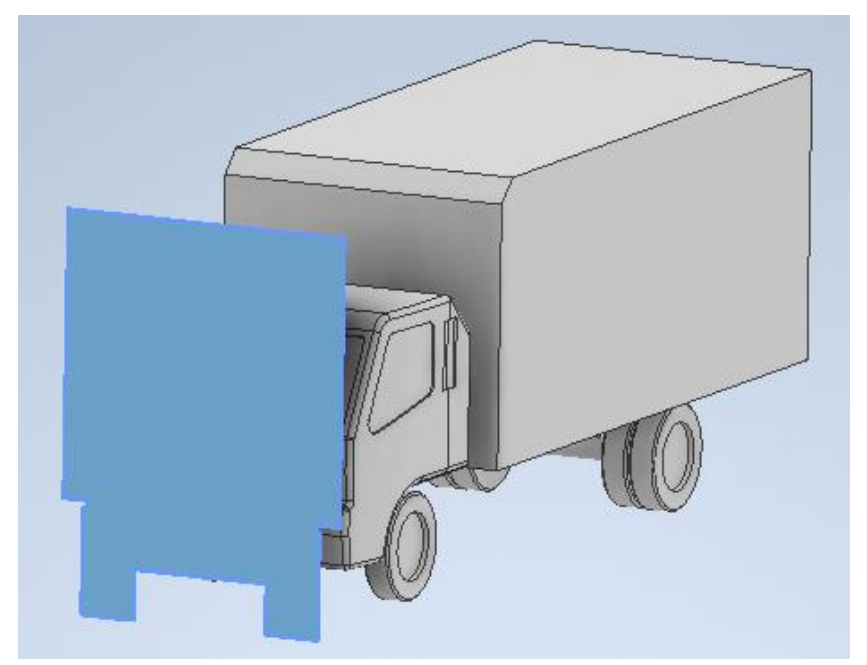

Figura 4. Diseño simplificado del camión

Fuente: Elaboración propia

La fuerza de arrastre varía según la velocidad que imprima el vehículo en carretera, por tanto, el rango de variación que se considera es de $0[\mathrm{~m} / \mathrm{s}]$ a $33.333[\mathrm{~m} / \mathrm{s}](120[\mathrm{~km} / \mathrm{h}])$, 
los otros valores de los parámetros que depende la ecuación unidimensional se indica en la tabla 3.

Tabla 3. Parámetros de la fuerza de arrastre

\begin{tabular}{lc}
\hline \multicolumn{1}{c}{ Descripción } & Valor \\
\hline Coeficiente aerodinámico & 0.78 (sin carenado superior) \\
(SAE J2188) & 0.64 (con carenado superior) \\
Densidad del aire & $1.1348714 \mathrm{~kg} / \mathrm{m}^{3}$ \\
Velocidad del aire & $1.4 \mathrm{~m} / \mathrm{s}$ \\
Área frontal & $8.223 \mathrm{~m}^{2}$ \\
\hline
\end{tabular}

Fuente: Elaboración propia

Se utiliza los datos anteriores en la ecuación unidimensional de la fuerza de arrastre, dando como resultado los valores registrados en la tabla 4.

Tabla 4. Fuerza de arrastre

\begin{tabular}{cccc}
\hline $\begin{array}{c}\text { Velocidad } \\
\text { camión } \\
(\mathbf{k m} / \mathbf{h})\end{array}$ & $\begin{array}{c}\text { Velocidad } \\
\text { camión } \\
(\mathbf{m} / \mathbf{s})\end{array}$ & $\begin{array}{c}\text { Fuerza } \\
\text { arrastre }(\mathbf{k N}) \\
\text { sin carenado }\end{array}$ & $\begin{array}{c}\text { Fuerza } \\
\text { arrastre }(\mathbf{k N}) \\
\text { con carenado }\end{array}$ \\
\hline 0 & 0.000 & 0.007 & 0.006 \\
10 & 2.778 & 0.064 & 0.052 \\
20 & 5.556 & 0.176 & 0.145 \\
30 & 8.333 & 0.345 & 0.283 \\
40 & 11.111 & 0.570 & 0.468 \\
50 & 13.889 & 0.851 & 0.698 \\
60 & 16.667 & 1.188 & 0.975 \\
70 & 19.444 & 1.582 & 1.298 \\
80 & 22.222 & 2.031 & 1.667 \\
90 & 25.000 & 2.537 & 2.082 \\
100 & 27.778 & 3.099 & 2.543 \\
110 & 30.556 & 3.717 & 3.050 \\
120 & 33.333 & 4.391 & 3.603 \\
\hline
\end{tabular}

Fuente: Elaboración propia 
El valor de la fuerza de arrastre es mínimo $(0.007 \mathrm{kN})$ cuando el vehículo está detenido, esto ocurre por la acción que tiene la velocidad del viento $(1.4 \mathrm{~m} / \mathrm{s})$ sobre el vehículo, la variación de la curva se observa en la figura 5 , se utiliza las unidades de $[\mathrm{km} / \mathrm{h}]$ para la gráfica sin que esto afecte los datos obtenidos.

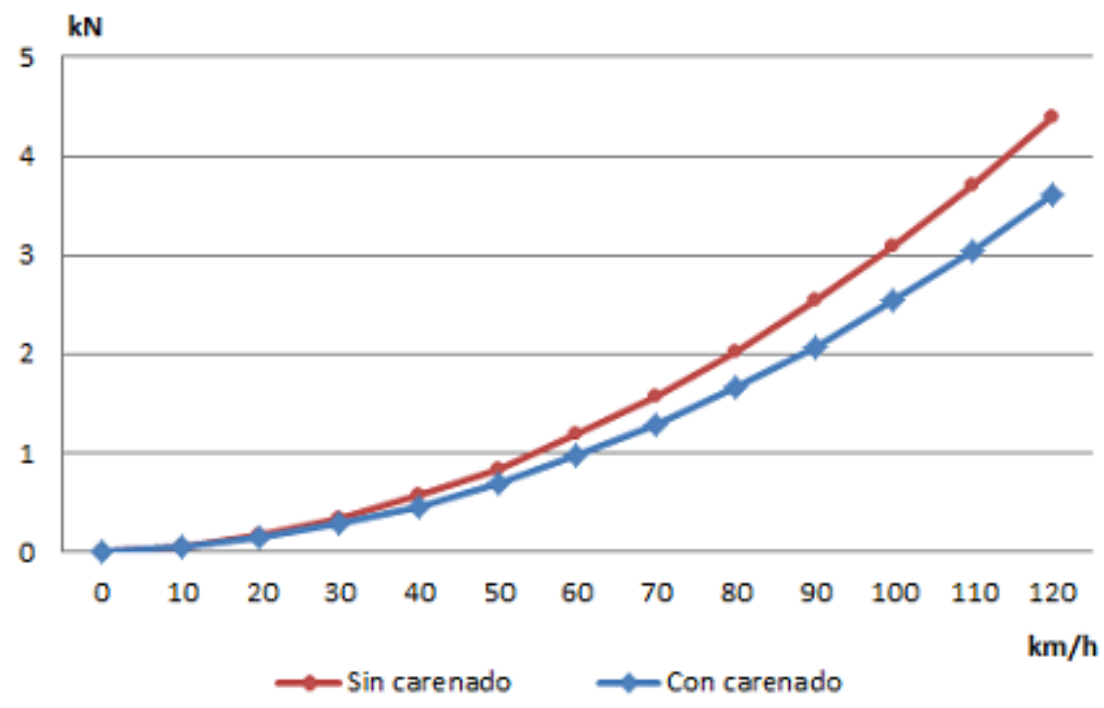

Figura 5. Fuerza de arrastre

Fuente: Elaboración propia

El límite máximo para la velocidad del transporte de carga en vía urbana es de 40 [km/h], la diferencia en la fuerza de arrastre $0.102[\mathrm{kN}]$, en relación a una recta en carretera que el límite es de $70[\mathrm{~km} / \mathrm{h}]$ adquiriendo una diferencia de $0.284[\mathrm{kN}]$, las normas SAE J1321 y DIN 70 030-2 que determinan consumo de combustible en camiones establecen una velocidad de prueba de $90[\mathrm{~km} / \mathrm{h}]$, la diferencia de fuerza de arrastre es de $0.455[\mathrm{kN}]$.

A mediados del mes de enero del 2020 el INAMHI emite un boletín meteorológico de advertencia, debido a ráfagas de viento que superan los $13.889[\mathrm{~m} / \mathrm{s}]$ que representa un nivel de amenaza medio (INAMHI, 2020).

Se utiliza el valor de 13.889 [m/s] en la velocidad del aire, obteniendo otros valores de la fuerza de arrastre, como se indica en la tabla 5.

Tabla 5. Fuerza de arrastre

\begin{tabular}{|c|c|c|c|}
\hline $\begin{array}{c}\text { Velocidad } \\
\text { camión } \\
(\mathbf{k m} / \mathbf{h})\end{array}$ & $\begin{array}{c}\text { Velocidad } \\
\text { camión } \\
(\mathrm{m} / \mathrm{s})\end{array}$ & $\begin{array}{c}\text { Fuerza } \\
\text { arrastre }(\mathbf{k N}) \\
\text { sin carenado }\end{array}$ & $\begin{array}{c}\text { Fuerza } \\
\text { arrastre }(k \mathbf{k}) \\
\text { con carenado }\end{array}$ \\
\hline 0 & 0.000 & 0.702 & 0.576 \\
\hline 10 & 2.778 & 1.011 & 0.830 \\
\hline 20 & 5.556 & 1.376 & 1.129 \\
\hline
\end{tabular}




\begin{tabular}{cccc}
30 & 8.333 & 1.797 & 1.475 \\
40 & 11.111 & 2.275 & 1.866 \\
50 & 13.889 & 2.808 & 2.304 \\
60 & 16.667 & 3.398 & 2.788 \\
70 & 19.444 & 4.044 & 3.318 \\
80 & 22.222 & 4.746 & 3.894 \\
90 & 25.000 & 5.504 & 4.516 \\
100 & 27.778 & 6.319 & 5.184 \\
110 & 30.556 & 7.189 & 5.899 \\
120 & 33.333 & 8.116 & 6.659 \\
\hline
\end{tabular}

Fuente: Elaboración propia

Se ha generado un aumento considerable del valor de la fuerza de $0.007[\mathrm{kN}]$ a 0.702 $[\mathrm{kN}]$ cuando el vehículo se encuentre detenido, solo por la acción del viento. El carenado superior comienza a trabajar desde este caso, con una diferencia de $0.126[\mathrm{kN}]$, a una velocidad del vehículo de $40[\mathrm{~km} / \mathrm{h}$ ] en vía urbana la diferencia es 0.409 [kN], en una recta en carretera se tiene una velocidad de $70[\mathrm{~km} / \mathrm{h}]$ con una diferencia de $0.726[\mathrm{kN}]$ y a una velocidad de prueba $90[\mathrm{~km} / \mathrm{h}]$ se tiene una diferencia de $0.988[\mathrm{kN}]$, esto se indica en la figura 6.

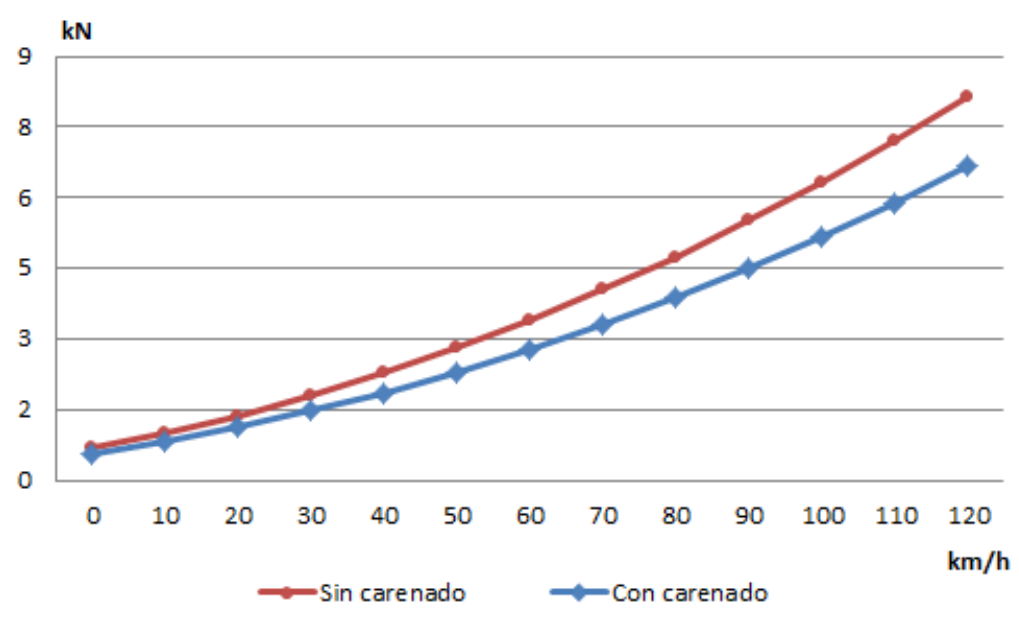

Figura 6. Fuerza de arrastre

Fuente: Elaboración propia

Se utiliza el módulo de CFX de ANSYS, para ello se requiere de una geometría simplificada a escala real, se realiza el dominio (túnel de viento) por donde circula el flujo de aire, para lo cual se considera un tamaño adecuado por las horas de simulación y restricciones innecesarias generadas por el propio dominio hacia el objeto de análisis. El mallado realizado consta de 303419 nodos y 1635059 elementos, el dominio y el mallado se indican en la figura 7 . 

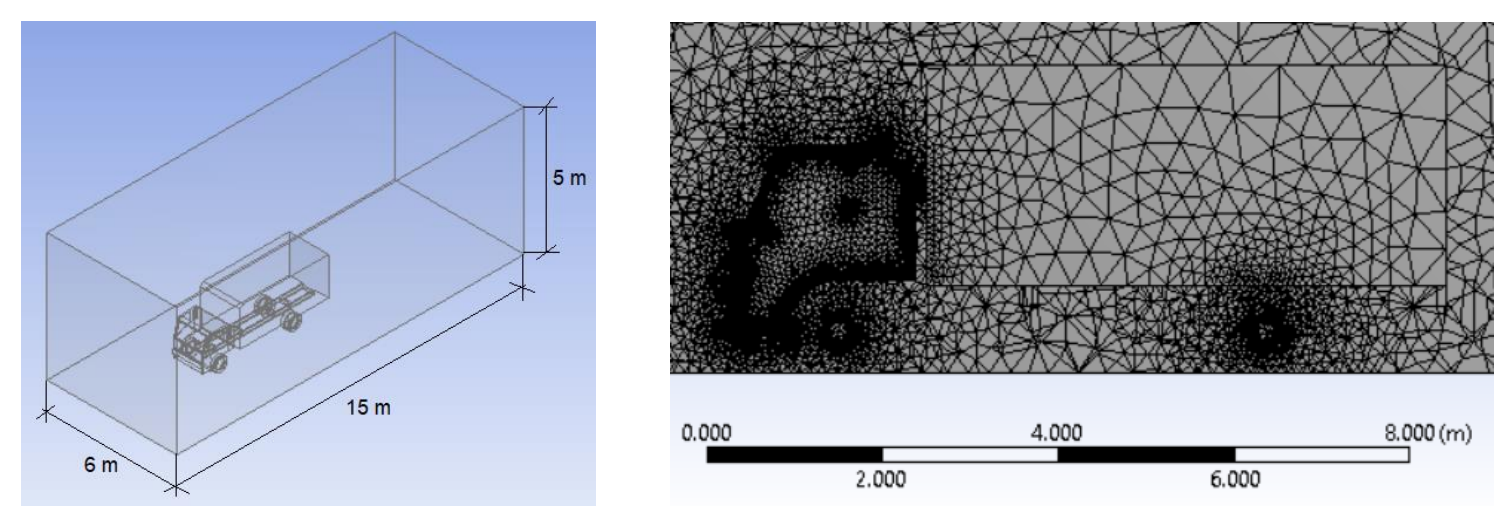

Figura 7. Dominio y mallado

Fuente: Elaboración propia

Las condiciones de frontera utilizadas en la simulación corresponden a un ingreso de aire a $25\left[{ }^{\circ} \mathrm{C}\right]$, con una presión atmosférica de 1 atm y una velocidad de $120[\mathrm{~km} / \mathrm{h}]$, el dominio y la superficie del vehículo tienen una condición de pared deslizante libre.

El flujo de aire choca contra el vehículo deteniéndose en la parte frontal y superior de la cabina, zona inferior del vehículo y la parte posterior de la zona de carga, la variación de la velocidad es de 0.04 [m/s] a 80.80 [m/s] como se indica en la figura 8 , en ambos casos la velocidad máxima ocurre en las aristas vivas que posee el vehículo, de manera global la velocidad del aire se debe mantener a lo largo del vehículo, como se observa en la parte superior de la cabina, esta restricción se corrige con el carenado superior.

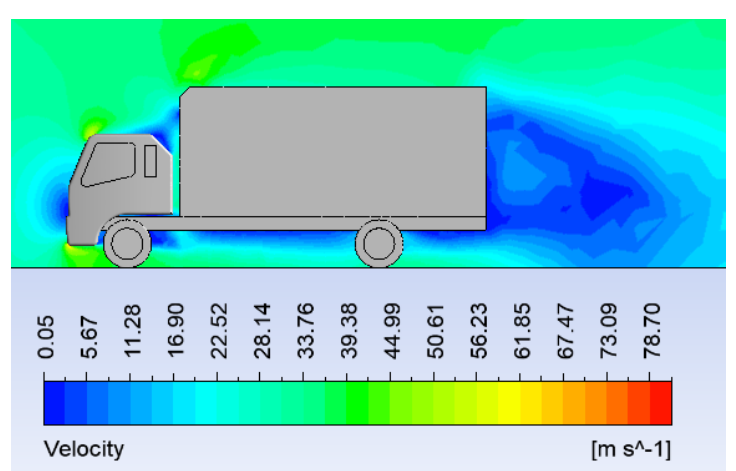

a) Sin carenado superior

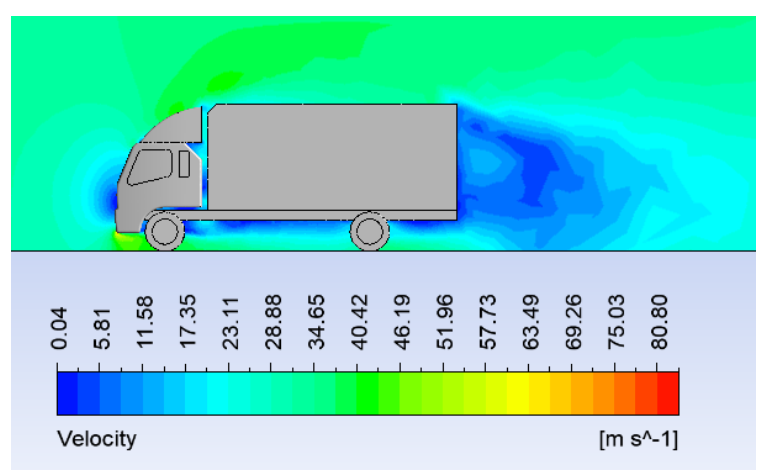

b) Con carenado superior

Figura 8. Velocidad del aire (plano 2D) en CFD

Fuente: Elaboración propia 
El flujo de las líneas de corriente del aire choca contra el vehículo y se desplazan a lo largo del vehículo con se observa en la figura 9.

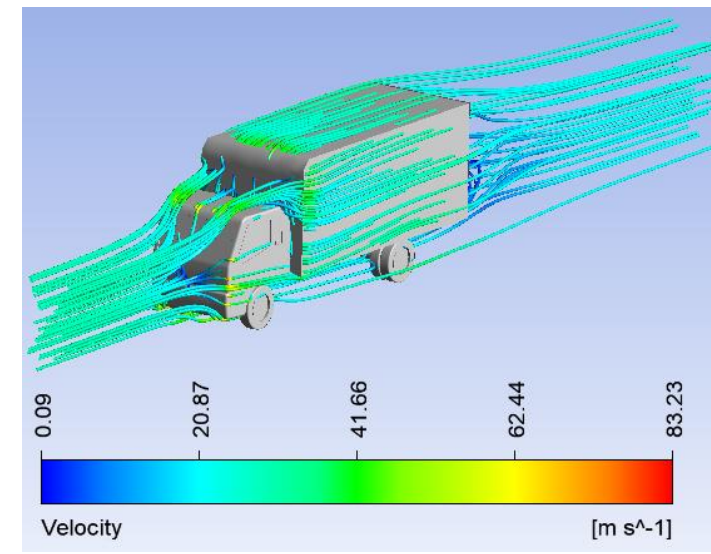

a) Sin carenado superior

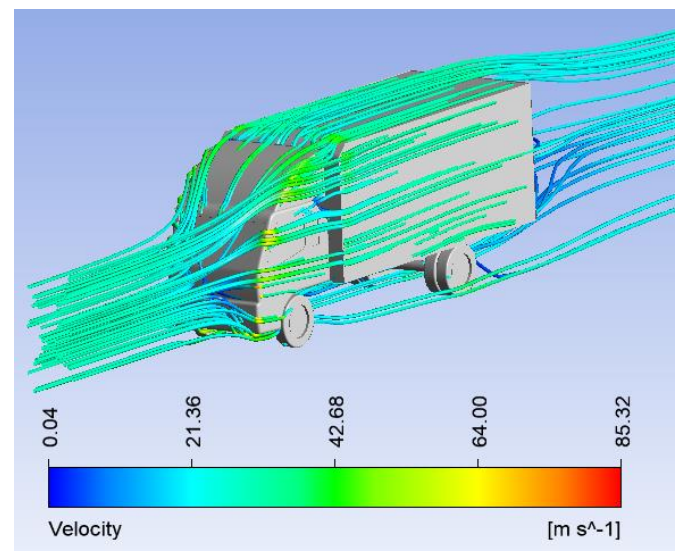

b) Con carenado superior

Figura 9. Velocidad del aire 3D en CFD

Fuente: Elaboración propia

El área frontal del vehículo recibe el primer contacto con el flujo de aire, de manera que se genera una presión máxima de 102292 [Pa] como se indica en la figura 10a, esto es debido a que el aire choca y disminuye su velocidad, acumulándose en estas zonas generando la presión, con la utilización del carenado superior se reduce esta presión en la zona de carga como se indica en la figura $10 \mathrm{~b}$.

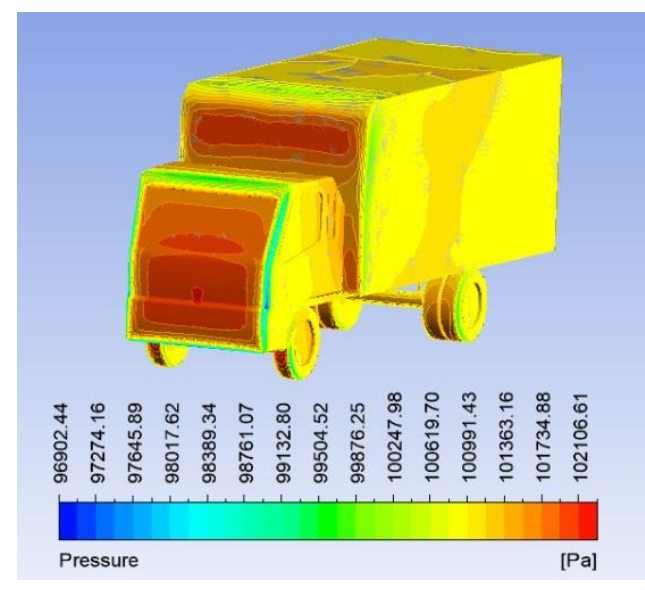

a) Sin carenado superior

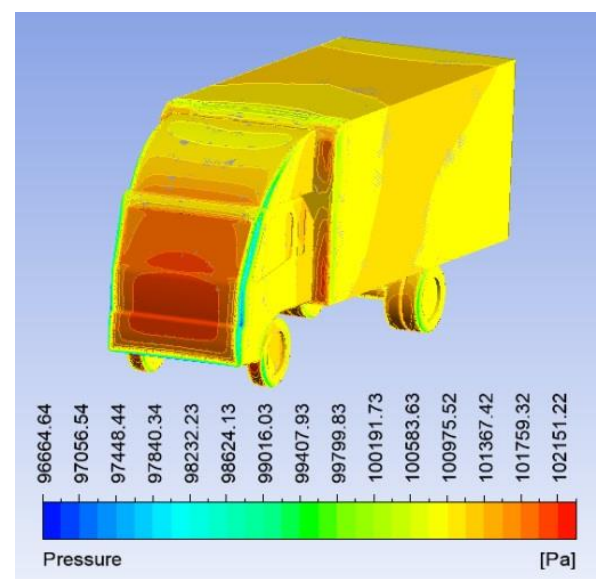

b) Con carenado superior

Figura 10. Presión del aire en CFD

Fuente: Elaboración propia 
El software CFX estima la fuerza de arrastre con un valor de 4.807 [kN] sin el carenado superior y un valor de $4.387[\mathrm{kN}]$ con el carenado superior, la diferencia es $0.42[\mathrm{kN}]$ que representa el 8.734 [\%] de reducción. En un semi-trailer se consigue una reducción del 17.6 [\%] en la fuerza de arrastre utilizando un carenado superior y carenados laterales.

El ahorro de consumo de combustible diésel bajo la norma DIN 70 030-2 en el corredor vial estatal Santo Domingo - La Concordia es de 4.63 [\%]. Estudios en Chile bajo la norma SAE J1321 demuestran un ahorro del 3.96 [\%] en el consumo de combustible al comparar el diseño de la cabina del camión y otro estudio un valor de 15.4 [\%] utilizando varios dispositivos aerodinámicos.

\section{Conclusiones.}

- Mediante un software de dinámica de fluidos computacional se estima un valor de 8.734 [\%] de reducción en la fuerza de arrastre al utilizar un carenado superior de cabina, derivando en un ahorro en el consumo de combustible de 4.63 [\%] en carretera. Para esto se toma en cuenta las condiciones meteorológicas de la zona, características del camión conjuntamente con su estructura de carga y carenado superior de cabina.

- La fuerza restrictiva generada por el aire se incrementa con la velocidad que adquiere el vehículo, esta fuerza actúa incluso si el vehículo se encuentra detenido, debido a la acción de la velocidad del viento que choca contra el vehículo, en menor o mayor cantidad dependiendo de la ubicación geográfica dónde se encuentre. La importancia radica en el gran número de unidades de transporte de carga que circulan por las carreteras de las diferentes regiones litoral, sierra y amazonia.

- La gran variedad de dispositivos aerodinámicos (carenado superior y lateral de cabina, cubre tanque, tapa cubos; carenado frontal, lateral y posterior de remolque; generador de vórtices, etc.) requieren un estudio previo para su implementación en el vehículo, ya que estos accesorios son fabricados bajo especificaciones de un determinado vehículo. La adición de varios de estos implementos podría generar mejores resultados.

\section{Referencias bibliográficas.}

AEADE. (2019). Anuario. Quito: AEADE. 
Aguilar, Y., Caldas, I., Rivera, A., \& Tapia, E. (2017). Estudio de la influencia de la apertura de las ventanas y la velocidad de circulación en la aerodinámica de un vehículo turismo. Cuenca: INGENIUS.

Ansys. (2018). CFX - Help support. ANSYS, Inc.

Balance-Energético. (2016). Balance Energético Nacional. Quito: Ministerio de Energías y recursos naturales no renovables.

Bayindirli, C., Salman, M., \& Akansu, Y. (2016). The Determination of Aerodynamic Drag Coefficient of Truck and Trailer Model by Wind Tunnel Tests. Turkey: Academicpaper.

CENAM. (21 de 06 de 2020). Cálculo de la densidad del aire utilizando la formula del CIPM-2007. Obtenido de http://www.cenam.mx/publicaciones/cdensidad.aspx

Ehsani, M., Gao, Y., Gay, S., \& Emadi, A. (2014). Vehículos Modernos Eléctricos, Híbridos Eléctricos y de Celdas de Combustible. USA: CRC PRES.

Hino-Motors. (2014). Especificaciones Hino 500 modelo GD8JLSA. Japon: Hino Motors, Ltd.

Hirsch, C. (2007). Fundamentos de dinámica computacional de fluidos. India: JohnWiley $\&$ Sons.

Hirz, M., \& Stadler, S. (2013). A new approach for the reduction of aerodynamic drag of long-distance transportation vehicles. USA: SAE.

INAMHI. (2020). Boletín meteorológico $N^{\circ}$ 03. Quito: INAMHI.

INAMHI. (28 de 06 de 2020). Red de estaciones automáticas hidrometeorológicas . Recuperado el 21 de 07 de 2020, de http://186.42.174.236/InamhiEmas/\#

Jhon D., A. (1991). Fundamentos de Aerodinámica. USA: McGraw-Hill.

Pachacama, D., \& Simbaña, J. (2017). Evaluación del consumo de combustible de un camión con la implementación de un deflector de aire. Quito: EPN.

Petroecuador. (2019). Informe estadístico anual . Quito: EP Petroecuador.

Remache, A., Tipanluisa, L., Salvador, J., \& Erazo, W. (2015). Análisis aerodinámico regional mediante cfd de un semirremolque cisterna para transporte de cemento. Perú: UNASAM.

SAE-J2188. (2018). Commercial Truck and Bus SAE Recommended Procedure for Vehicle Performance Prediction and Charting. USA: SAE.

Villalobos, J., Arancibia, N., Retamal, S., Olivo, P., \& Vásquez, J. (2011). Impacto de la aerdinámica para el ahorro de combustible. Chile: AChEE. 
Villalobos, J., Gavilan, C., Salazar, C., \& Rojas, C. (2012). Impacto del diseño de cabinas en el consumo de combsutible. Chile: AChEE.

\section{Ligital}




\section{PARA CITAR EL ARTÍCULO INDEXADO.}

Pachacama Gualotuña, D. P., Pachacama Gualotuña, D. A., Córdova Orellana, C. C., \& Llulluna Llumiquinga, F. R. (2021). Influencia de un carenado superior en la fuerza de arrastre de un camión . Ciencia Digital, 5(2), 109-126. https://doi.org/10.33262/cienciadigital.v5i2.1610

\section{\Ciencia}

El artículo que se publica es de exclusiva responsabilidad de los autores y no necesariamente reflejan el pensamiento de la Revista Ciencia Digital.

El artículo queda en propiedad de la revista y, por tanto, su publicación parcial y/o total en otro medio tiene que ser autorizado por el director de la Revista Ciencia Digital.
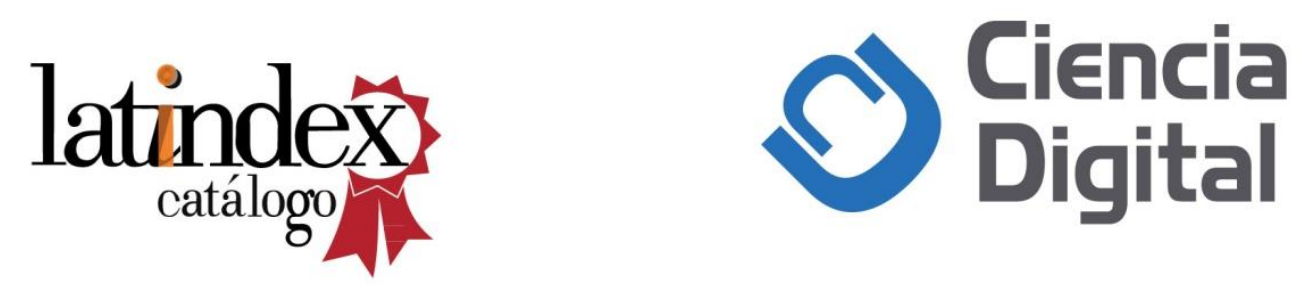